\title{
Elevated preoperative heart rate associated with increased risk of cardiopulmonary complications after resection for lung cancer
}

\author{
Danxia Fu, Chaoshuang Wu, Xiaoyu Li and Junping Chen * (1)
}

\begin{abstract}
Background: The study aimed to assess whether preoperative resting heart rate could be a risk factor for cardiopulmonary complications (CPCS) after lung cancer resection.

Methods: Eligible consecutive patients who underwent resection surgery for non-small cell lung cancer (NSCLC) at Ningbo NO.2 Hospital between May, 2010 and July, 2015 were included. The demographic, clinical characteristics and laboratory parameters were compared in patients with or without CPCs within postoperative 30 days. The multivariate logistic regression analysis was used to analyze the association between CPCs and risk factors. Receiver operating characteristic (ROC) curve analysis was utilized for the predictive role of preoperative resting heart rate for CPCs.

Results: One hundred eighty participants were enrolled into the final analysis and 42 of them had an established diagnosis of CPCS within postoperative 30 days. Elevated preoperative resting heart rate was an independent risk factor for postoperative CPCs (OR: $4.48,95 \%$ Cl: 1.17-18.42, $P=0.021$ ) by the multivariate logistic regression analysis. ROC curve analysis indicated elevated resting heart rate as a predictor for CPCs with a cut-off value of 86 beats/min (AUC: 0.813 , specificity: $80.95 \%$, sensitivity: $72.46 \%, P<0.001$ ).

Conclusions: Elevated preoperative resting heart rate was associated with an increased risk of postoperative CPCs in patients after resection for lung cancer.
\end{abstract}

Keywords: Resting heart rate, Cardiopulmonary complications, Risk assessment, Lung cancer

\section{Background}

Lung resection is the standard therapeutic strategy for early-stage lung cancer management and it provides the best chance of cure [1]. Lung resection can always result in lung function impairment, a higher incidence of postoperative complications and mortality in comparison with some other surgical disciplines [2]. Previous data has reported a mortality rate for lung resection surgery as high as $6.7 \%$ and cardiopulmonary complications (CPCs) are major causes for postoperative mortality and morbidity [3]. Therefore, appropriate preoperative management and valid CPCs prediction are of great importance in minimizing the morbidity or mortality.

\footnotetext{
* Correspondence: yunfeiyangtg@126.com

Department of anesthesiology, Ningbo NO.2 hospital, NO.41 Xibei Street, Ningbo City 315000, Zhejiang Province, China
}

Pulmonary function and cardiopulmonary fitness measurement have been suggested for patient risk stratification $[4,5]$. Some other parameters such as gender, body mass index (BMI), renal dysfunction, etc. are also suggested as risk factors for CPCs [6]. A close association has been demonstrated between resting heart rate and an elevated incidence of cardiovascular mortality both in patients with cardiac diseases and general population [7]. Previous data have indicated the predictive value of increased heart rate for unfavorable outcomes in those patients with an acute coronary syndrome or heart failure [8]. Recent studies have revealed that elevated preoperative resting heart rate ( $>87$ beats $/ \mathrm{min}$ ) significantly correlates with postoperative myocardial impairment and mortality in patients undergoing non-cardiac surgery [9].

(c) The Author(s). 2018 Open Access This article is distributed under the terms of the Creative Commons Attribution 4.0 International License (http://creativecommons.org/licenses/by/4.0/), which permits unrestricted use, distribution, and reproduction in any medium, provided you give appropriate credit to the original author(s) and the source, provide a link to the Creative Commons license, and indicate if changes were made. The Creative Commons Public Domain Dedication waiver (http://creativecommons.org/publicdomain/zero/1.0/) applies to the data made available in this article, unless otherwise stated. 
To reduce selection bias, only one type of surgery (lung cancer) was chosen in this study. In comparison with other non-cardiac surgery, thoracic surgery shows a higher morbidity rate of CPCs. Due to the high morbidity rate and mortality, establishing an association between heart rate and CPCs is of great importance for risk stratification. However, few data has specifically explored the relationship between heart rate and postoperative CPCs in patients with lung cancer. The current study aimed to assess potential risk factors for the CPCs after resection for lung cancer.

\section{Methods}

\section{Patients}

This study was approved by the Medical Institutional Ethics Committee of Zhejiang province. We prospectively reviewed eligible consecutive patients who underwent resection surgery for non-small cell lung cancer (NSCLC) at Ningbo NO.2 Hospital between May, 2010 and July, 2015. Patients enrolled were required to provide written informed consent before the study.

\section{Data collection}

Demographics data were collected preoperatively set from patients and their medical records including age, gender, body mass index (BMI), smoking habits, American Society of Anesthesiologists (ASA) physical status, preoperative pre-existing cardiovascular medications, etc. The preoperative comorbidities including diabetes, hypertension, chronic obstructive pulmonary disease (COPD), coronary artery disease (CAD), chronic kidney disease (CKD), prior myocardial infarction and atrial fibrillation were also recorded in details.

All participants enrolled were required to undergo electrocardiograms, pulmonary function tests and blood gas analysis before the operation. Preoperative evaluation of Revised Cardiac Risk Index (RCRI) and cardiovascular comorbidities were according to the updated American College of Cardiology (ACC)/American Heart Association (AHA) Task Force guidelines [10]. Those patients with cardiac risks or diseases (cardiac surgery history, ischemic heart disease, etc.) underwent full physical evaluation by an experienced cardiologist. If necessary, a further echocardiographic test or invasive evaluation examinations was conducted to assess their cardiac status deeply. The clinical data including tumor location, clinical stage, nodal stage and types of surgery were also documented according to the observations during operation and postoperative pathological results. Baseline resting heart rate was measured using a digital monitor after patients have sat and rested for at least $5 \mathrm{~min}$. Readings were taken for three times and the mean value was recorded.
The primary end point of this study was CPCs within postoperative 30 days.

\section{Definition of CPCs}

The definition of postoperative CPCs was according to the descriptions by Duc et al. [11], including pleural effusion requiring drainage, mechanical ventilation within postoperative $48 \mathrm{~h}$, atelectasis, acute respiratory distress syndrome (ARDS), respiratory failure, pneumonia, pneumothorax, pulmonary embolism, empyema, ventricular or atrial arrhythmias, myocardial infarction.

\section{Laboratory tests}

Fasting blood samples were obtained in the morning on 1 day prior to the operation from all participants. The concentrations of cardiac troponin $\mathrm{T}$ (cTnT) and inflammatory cytokines including C-reactive protein (CRP) and tumor necrosis factor- $\alpha$ (TNF- $\alpha$ ) were measured using enzyme-linked immunesorbent assays (ELISA) according to the manufacturers' instructions (R\&D Systems, Minneapolis, MN, USA). The expressions of hemoglobin, albumin, creatinine and urea were also measured from the blood samples.

\section{Statistical analysis}

SPSS 19.0 (SPSS, Inc.) and Graphpad 5.0 (Instat, San Diego, CA) were used for statistical analysis in our study. A sample size calculation was performed before the study and at least 138 patients would be required using a $5 \%$ significance level and $80 \%$ power. We used the estimated incidence of CPCs as a basis for the sample size estimation according to the reference values reported and our previous clinical experience. Data are given as number (n) with percentage (\%), or mean \pm standard error (SD). Chi-squared test or Fisher's exact test was used for the statistical analysis of categorical variables. Numeric variables were analyzed by unpaired $t$ test (for normal distribution) or Mann Whitney test (for non-normal distribution). We used multivariate logistic regression analysis to analyze for association between $\mathrm{CPCs}$ and risk factors. In the logistic regression model, the categorical data (including CRP, TNF- $\alpha$, etc.) were grouped into high group vs low group with the median level as the cut-off value. Receiver operating characteristic (ROC) curve analysis was utilized for the predictive role of resting heart rate for CPCs. All statistical tests were bilateral probability and a $P$ value of $<0.05$ was accepted as statistically significant.

\section{Results}

\section{Patient characteristics}

Two hundred three participants were recruited into this study between May, 2010 and July, 2015. After excluding 23 patients with missing data or refusal of cooperation, 
the remained 180 NSCLC patients were enrolled into the final analysis. The mean age of participants was 62.6 years and $88(48.9 \%)$ were male patients. Of all the 180 NSCLC patients, 106 (58.9\%) were squamous cell carcinoma, 65 (36.1\%) were adenocarcinoma and 9 (5.0\%) were mixed. The detailed patient characteristics are presented in Tables 1 and 42 of the 180 (23.3\%) had an established diagnosis of CPCs within postoperative 30 days. Pleural effusion (15/180, 8.3\%) and arrhythmias $(11 / 180,6.1 \%)$ were the two most common complications in this series, which is shown in Table 1. There were no statistical differences between patients in $\mathrm{CPCs}$ group and non-CPCs group with respect to gender, ASA physical status, BMI, smoking habits, tumor location, nodal stage, pulmonary function test and type of surgery $(P>0.05)$. Before the surgery, the pre-existing cardiovascular medications in patients with or without CPCs did not differ significantly except beta-blocker. Preoperative comorbidities including hypertension and coronary artery disease (CAD) were more frequently observed in patients with CPCs $(P<0.05)$. The percentage of prior cardiothoracic surgery history was similar for patients with CPCs compared with those without CPCs $(P>0.05)$. Patients who suffered CPCs showed a significantly higher resting heart rate, RCRI score and clinical stage than those without CPCs $(P<0.05)$.

\section{Laboratory tests}

As illustrated in Table 2, laboratory parameters were compared between patients with or without CPCs. Patients with CPCs exhibited higher expressions of cTnT, CRP and TNF- $\alpha(P<0.05)$.

\section{Risk factors for CPCs}

All the potential risk factors associated with CPCs mentioned above were enrolled into the final multivariate logistic regression analysis. As shown in Table 3, elevated preoperative resting heart rate was an independent risk factor for postoperative CPCs during patients after resection for lung cancer (OR: 4.48, 95\% CI: 1.17-18.42, $P=0.021)$.

\section{Predictive role of resting heart rate for CPCs}

The predictive role of resting heart rate for CPCs was analyzed by ROC curve analysis. An elevated resting heart rate was a predictor for CPCs with a cut-off value of 86 beats/min (AUC: 0.813 , specificity: $80.95 \%$, sensitivity: $72.46 \%, P<0.001$, see Fig. 1 ).

\section{Discussion}

As the best cure strategy in patients with resectable NSCLC, surgery is still associated with a high complication rate [12]. CPCs are reported to be a major source of morbidity and mortality in the acute phase after surgery [13]. Previous studies performed in patients after lung cancer surgery have revealed that CPCs, with a high prevalence ranged $20-35 \%$, have a profound effect on both short-term and long-term outcomes [13, 14]. The principal finding of the current single-center cohort study was that an elevated preoperative resting heart rate (cut-off value: 86 beats $/ \mathrm{min}$ ) was associated with an increased incidence of CPCs in patients after resection for lung cancer. The current study was the first to identify the possible relationship between preoperative resting heart rate and CPCs within 30 days.

Previous studies have hypothesized that a preoperative resting heart rate over 87 beats/min is significantly associated with impaired autonomic, cardiovascular function and subclinical cardiac failure [9], which is quite consistent with our results. Preoperative diagnosis of cardiac failure is closely related to increased morbidity and mortality in patients undergoing non-cardiac surgery [15]. Furthermore, a close association between increased heart failure and progressed resting heart rate has also been exposed in elderly healthy participants [16]. Previous studies have indicated that advanced age, elevated baseline heart rate, increased serum expressions of glucose, albumin and creatinine are all independent predictors of incident heart failure in elderly patients [17], which is in accordance with our results. Increasing evidence has indicated that preoperative usage of beta-blockade is associated with improved outcomes in patients at high risk of cardiac events after non-cardiac surgery [18]. Furthermore, the preoperative beta-blockade withdrawal is associated with an increased mortality and risk of postoperative adverse cardiac events in vascular surgical patients [19]. However, in contrast, another review by Giles et al. indicates that chronic medication therapy of beta-blockade correlates with an increased risk of myocardial infarction in a surgical population [20]. In contrast, our results showed no predictive value for beta blockade medication on postoperative CPCs.

It has been well-understood that elevated heart rate with stable coronary artery stenosis impairs the distribution of subendocardial and epicardial blood flow with the reason that shortened diastolic time interval leads to myocardial dysfunction and subendocardial ischemia $[21,22]$. Numerous evidence is in support of the consistent association between perioperative heart rate and cardiovascular mortality in patients with high-risk cardiac events [23]. Recent studies have demonstrated the predictive value of intraoperative tachycardia for adverse outcomes including higher ICU admission, prolonged hospital stay and increased mortality in patients with major non-cardiac surgeries [24]. Heart rate has also been indicated as a significant predictive factor for overall 
Table 1 Demographics and clinical data of patients with or without CPCs

\begin{tabular}{|c|}
\hline Patient characteristics \\
\hline Age (year) \\
\hline Gender, n (\%) \\
\hline Male \\
\hline Female \\
\hline ASA physical status, n (\%) \\
\hline$\|$ \\
\hline III \\
\hline IV \\
\hline BMI $\left(\mathrm{kg} / \mathrm{m}^{2}\right)$ \\
\hline Current smoker, n (\%) \\
\hline Preoperative medications, n (\%) \\
\hline Beta-blocker \\
\hline Corticosteroid \\
\hline Calcium channel antagonist \\
\hline ACEI or ARB \\
\hline Diuretic \\
\hline Anti-platelet \\
\hline Statin \\
\hline Resting heart rate (beats/min) \\
\hline Prior cardiothoracic surgery, n (\%) \\
\hline Comorbidities, n (\%) \\
\hline Diabetes \\
\hline Hypertension \\
\hline COPD \\
\hline CAD \\
\hline CKD \\
\hline
\end{tabular}

Prior myocardial infarction

Atrial fibrillation

RCRI, n (\%)

II

III

Pathological type, n (\%)

Squamous cell carcinoma

Adenocarcinoma

Mixed

Tumor location, n (\%)

Upper

Middle

Lower

Clinical stage, n (\%)

I-II

III-IV

\begin{tabular}{lll}
$\begin{array}{l}\text { CPCs } \\
(n=42)\end{array}$ & $\begin{array}{l}\text { Non-CPCs } \\
(n=138)\end{array}$ & $P$-value \\
\hline $66.9 \pm 9.5$ & $61.3 \pm 8.3$ & $<0.01^{*}$
\end{tabular}

$23(54.8 \%) \quad 65(47.1 \%) \quad 0.385$

$19(45.2 \%) \quad 73(52.9 \%) \quad-$

$8(19.0 \%) \quad 36(26.1 \%) \quad 0.634$

$23(54.8 \%) \quad 71(51.4 \%)$

$11(26.2 \%) \quad 31(22.5 \%)$

$23.5 \pm 4.1 \quad 23.1 \pm 3.8 \quad 0.558$

$11(26.2 \%) \quad 32(23.2 \%) \quad 0.690$

$6(14.3 \%) \quad 42(30.4 \%) \quad 0.038^{*}$

$5(11.9 \%) \quad 11(8.0 \%) \quad 0.433$

$6(14.3 \%) \quad 26(18.8 \%) \quad 0.499$

$5(11.9 \%) \quad 22(15.9 \%) \quad 0.521$

$6(14.3 \%)$

$19(13.8 \%)$

0.932

$8(19.0 \%)$

$33(23.9 \%)$

$7(16.7 \%)$

$25(18.1 \%)$

0.510

$92.2 \pm 8.2$

$82.7 \pm 7.9$

0.830

$5(11.9 \%)$

$18(13.0 \%)$

$<0.01^{*}$

0.847

$7(16.7 \%)$

$18(13.0 \%)$

0.552

$14(33.3 \%)$

$24(17.4 \%)$

$0.027^{*}$

$14(33.3 \%)$

$32(23.2 \%)$

0.187

$12(28.6 \%)$

$20(14.5 \%)$

$0.037^{*}$

$2(4.8 \%)$

$7(5.1 \%)$

0.936

$6(14.3 \%)$

$15(10.9 \%)$

0.546

$7(16.7 \%)$

$19(13.8 \%)$

0.640

$0.033^{*}$

$15(35.7 \%)$

$72(52.2 \%)$

$12(28.6 \%)$

$42(30.4 \%)$

$15(35.7 \%) \quad 24(17.4 \%)$

$24(57.1 \%)$

$82(59.4 \%)$

$16(38.1 \%)$

$49(35.5 \%)$

$2(4.8 \%)$

7 (5.1\%)

$24(57.1 \%)$

$3(7.1 \%)$

$83(60.1 \%)$

$15(35.7 \%)$

$9(6.5 \%)$

$46(33.3 \%)$

$35(83.3 \%) \quad 129(93.5 \%)$

$7(16.7 \%) \quad 9(6.5 \%)$ 0.954
Table 1 Demographics and clinical data of patients with or without CPCs (Continued)

\begin{tabular}{|c|c|c|c|}
\hline Patient characteristics & $\begin{array}{l}\text { CPCs } \\
(n=42)\end{array}$ & $\begin{array}{l}\text { Non-CPCs } \\
(n=138)\end{array}$ & $P$-value \\
\hline Nodal stage, n (\%) & & & 0.189 \\
\hline No & $32(76.2 \%)$ & $121(87.7 \%)$ & - \\
\hline N1 & $7(16.7 \%)$ & $12(8.7 \%)$ & - \\
\hline N2 & $3(7.1 \%)$ & $5(3.6 \%)$ & - \\
\hline \multicolumn{4}{|l|}{ Pulmonary function test } \\
\hline Predicted FVC & $92.5 \pm 12.6$ & $92.6 \pm 13.1$ & 0.965 \\
\hline Predicted FEV1 & $79.3 \pm 18.9$ & $82.0 \pm 20.2$ & 0.443 \\
\hline Type of surgery, n (\%) & & & 0.613 \\
\hline Segmentectomy & $6(14.3 \%)$ & $19(13.8 \%)$ & - \\
\hline Pneumonectomy & 7 (16.7\%) & $15(10.9 \%)$ & - \\
\hline Lobectomy & $25(59.5 \%)$ & $82(59.4 \%)$ & - \\
\hline Bilobectomy & $4(9.5 \%)$ & $22(15.9 \%)$ & - \\
\hline \multicolumn{4}{|l|}{ Breakdown of CPCs, n (\%) } \\
\hline Pleural effusion & $15(8.3 \%)$ & - & - \\
\hline Arrhythmias & $11(6.1 \%)$ & - & - \\
\hline Pneumonia & $6(3.3 \%)$ & - & - \\
\hline Atelectasis & $3(1.7 \%)$ & - & - \\
\hline Respiratory failure & $2(1.1 \%)$ & - & - \\
\hline Pneumothorax & $2(1.1 \%)$ & - & - \\
\hline ARDS & $2(1.1 \%)$ & - & - \\
\hline Myocardial infarction & $1(0.6 \%)$ & - & - \\
\hline
\end{tabular}

CPCS cardiopulmonary complications, ASA American Society of Anesthesiologists, $B M I$ Body Mass Index, $A C E I$ angiotensin converting enzyme inhibitor, $A R B$ angiotensin receptor blocker, COPD chronic obstructive pulmonary disease, $C A D$, coronary artery disease, $C K D$ chronic kidney disease, $R C R I$ revised cardiac risk index, FVC forced vital capacity, FEV1 forced expiratory volume in 1 second, ARDS acute respiratory distress syndrome. $P$-values were calculated by Chi-square test, Fisher exact test or $t$ test. ${ }^{*} P$ value $<0.05$

Table 2 Preoperative laboratory tests of patients with or without CPCs

\begin{tabular}{llll}
\hline Laboratory tests & CPCs $(n=42)$ & Non-CPCs $(n=138)$ & $P$-value \\
\hline Hemoglobin(mg/dl) & $13.1 \pm 2.5$ & $12.9 \pm 3.0$ & 0.695 \\
Albumin $(\mathrm{g} / \mathrm{dL})$ & $3.9 \pm 0.4$ & $4.0 \pm 0.5$ & 0.238 \\
CTnT(ng/L) & $12.3 \pm 4.1$ & $9.7 \pm 2.8$ & $<0.01^{*}$ \\
CRP $(\mathrm{mg} / \mathrm{L})$ & $8.3 \pm 5.3$ & $6.1 \pm 4.1$ & $0.005^{*}$ \\
TNF-a(nmol/L) & $9.8 \pm 3.5$ & $7.7 \pm 2.5$ & $<0.01^{*}$ \\
Creatinine(mg/dL) & $1.0 \pm 0.4$ & $0.9 \pm 0.3$ & 0.083 \\
Urea(mmol/L) & $6.5 \pm 2.3$ & $5.9 \pm 2.5$ & 0.167
\end{tabular}

CPCS cardiopulmonary complications, CTnT cardiac troponin T, CRP C-reactive protein, TNF- $a$ tumor necrosis factor- $a$. $P$-values were calculated by $\mathrm{t}$ test or Mann-Whitney U-test. ${ }^{*} P$ value $<0.05$ 
Table 3 Preoperative risk factors associated with CPCs by multiple logistic regression analysis

\begin{tabular}{llll}
\hline Parameter & \multicolumn{2}{l}{ CPCs } & \\
\cline { 2 - 4 } & OR & $95 \% \mathrm{Cl}$ & $P$ value \\
\hline Age & 0.58 & $0.22-1.62$ & 0.13 \\
Beta-blocker & 0.94 & $0.88-1.03$ & 0.094 \\
Resting heart rate & 4.48 & $1.17-18.42$ & $0.021^{*}$ \\
Hypertension & 5.04 & $0.93-17.54$ & 0.087 \\
CAD & 0.38 & $0.13-1.17$ & 0.079 \\
RCRI & 0.95 & $0.92-1.01$ & 0.10 \\
Clinical stage (I/II X III/IV) & 0.89 & $0.73-1.04$ & 0.23 \\
CTnT & 0.92 & $0.81-1.08$ & 0.31 \\
CRP & 1.02 & $0.88-1.18$ & 0.84 \\
TNF-a & 1.09 & $0.82-1.48$ & 0.42
\end{tabular}

$C P C$ s cardiopulmonary complications, $C A D$ coronary artery disease, $R C R I$ revised cardiac risk index, $C T n T$ cardiac troponin T, CRP C-reactive protein, TNF- $a$ tumor necrosis factor-a. $\mathrm{Cl}$ : Confidence Interval, OR Odds Ratio. ${ }^{*} P$ value $<0.05$

survival during patients with septic shock [25]. Previous data have found an independent association between an increase of heart rate at 10 beats/min and an increased risk of cardiac failure at $11 \%$ [26].

Patients with higher preoperative heart rate are at increased risk of postoperative CPCs. The underlying mechanisms linking heart rate to $\mathrm{CPCs}$ still remain unclear, but some potential mechanisms may promote the CPCs risk in subjects after resection for lung cancer. Elevated heart rate links closely to coronary blood flow, myocardial injury and myocardial oxygen demand. The supply-demand imbalance of myocardial oxygen caused by elevated heart rate may be a possible explanation for the induction of postoperative

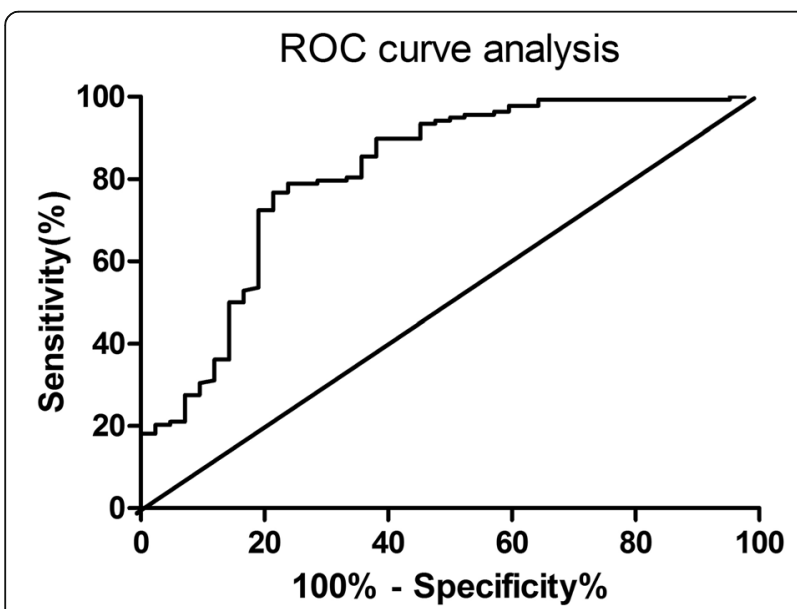

Fig. 1 ROC curve analysis of resting heart rate for CPCs in patients after resection for lung cancer. An elevated resting heart rate was a predictor for CPCs with a cut-off value of 86 beats/min (AUC: 0.813, specificity: $80.95 \%$, sensitivity: $72.46 \%, P<0.001)$. ROC, receive operating characteristic; CPCs, cardiopulmonary complications; AUC, area under curve
CPCs. As a result of lower stroke volume, heart rate is subsequently increased to maintain the cardiac output. Increased CPCs risk can be ascribed to the oxygen delivery, which is supported by consistent relationship between elevated heart rate and reduced $\mathrm{VO}_{2}$ [27].

We conclude that patients with an elevated preoperative heart rate before lung surgery may have an increased risk of postoperative CPCs. However, why higher preoperative heart rate is associated with increased complications after lung cancer surgery remains unclear. The surgeon and anesthetist can differentiate the patients according to the preoperative heart rate and treat them accordingly. Predicting CPCs helps the surgeon to evaluate the hospital stay, intensive care unit stay, economic costs, and outcomes. Furthermore, the surgeon can decide surgery plans as well as inoperability after physiological assessment. We recommend that those patients with preoperative heart rate (over 86 beats/min) can be categorized into high-risk surgical patients and individualized approaches to care are recommended.

We acknowledge that the study has the following study limitations. The sample size of this study was relatively small and involved mechanisms remained unclear. A long follow up and monitoring the incidence of CPCs would improve the precision and have better effects. Furthermore, we have to acknowledge that there were some residual and unmeasured confounding factors. At last, only the patients of certain disease (lung cancer) were enrolled, whether preoperative heart rate could be a more general predictor for prognosis than specifically for certain diseases remained unknown.

\section{Conclusions}

In conclusion, our results revealed that elevated preoperative resting heart rate was associated with an increased risk of postoperative CPCs in patients after resection for lung cancer.

\section{Abbreviations}

ACEl: Angiotensin converting enzyme inhibitor; ARB: Angiotensin receptor blocker; ASA: American Society of Anesthesiologists; BMI: Body Mass Index; CAD: Coronary artery disease; Cl: Confidence Interval; CKD: Chronic kidney disease; COPD: Chronic obstructive pulmonary disease; CPCs: Cardiopulmonary complications; CRP: C-reactive protein; CTnT: Cardiac troponin T; ELISA: Enzymelinked immunesorbent assays; FEV1: Forced expiratory volume in 1second; FVC: Forced vital capacity; OR: Odds Ratio; RCRI: Revised cardiac risk index; ROC: Receiver operating characteristic; SD: Standard error; TNF-a: Tumor necrosis factor-a

\section{Availability of data and materials}

The datasets used and analyzed during the current study are available from the corresponding author on reasonable request.

\section{Authors' contributions}

DX F and CS W participated in the conception and design, data collection, statistical analysis and wrote the manuscript. JP C and XY L participated in the conception and design and data collection. All authors read and approved the final manuscript. 


\section{Ethics approval and consent to participate}

This study was approved by the Medical Institutional Ethics Committee of Zhejiang province. Written informed consent was obtained from each patient enrolled in this study.

\section{Consent for publication}

Not applicable.

\section{Competing interests}

The authors declare that they have no competing interests.

\section{Publisher's Note}

Springer Nature remains neutral with regard to jurisdictional claims in published maps and institutional affiliations.

Received: 7 December 2017 Accepted: 12 July 2018

Published online: 25 July 2018

\section{References}

1. Howington JA, Blum MG, Chang AC, Balekian AA, Murthy SC. Treatment of stage I and II non-small cell lung cancer: diagnosis and management of lung cancer, 3rd ed: American College of Chest Physicians evidence-based clinical practice guidelines. Chest. 2013;143(5 Suppl):e278S-313S.

2. Toker A, Ziyade S, Bayrak Y, Tanju S, Senturk M, Dilege S, et al. Prediction of cardiopulmonary morbidity after resection for lung cancer: stair climbing test complications after lung cancer surgery. Thorac Cardiovasc Surg. 2007; 55(4):253-6.

3. Brunelli A, Kim AW, Berger Kl, Addrizzo-Harris DJ. Physiologic evaluation of the patient with lung cancer being considered for resectional surgery: diagnosis and management of lung cancer, 3rd ed: American College of Chest Physicians evidence-based clinical practice guidelines. Chest. 2013; 143(5 Suppl):e166S-e90S

4. Brunelli A, Charloux A, Bolliger CT, Rocco G, Sculier JP, Varela G, et al. ERS/ ESTS clinical guidelines on fitness for radical therapy in lung cancer patients (surgery and chemo-radiotherapy). Eur Respir J. 2009;34(1):17-41.

5. Lim E, Baldwin D, Beckles M, Duffy J, Entwisle J, Faivre-Finn C, et al. Guidelines on the radical management of patients with lung cancer. Thorax. 2010; 65(Suppl 3):iii1-27.

6. Kozower BD, Sheng S, O'Brien SM, Liptay MJ, Lau CL, Jones DR, et al. STS database risk models: predictors of mortality and major morbidity for lung cancer resection. Ann Thorac Surg. 2010;90(3):875-81. discussion 81-3

7. Palatini P. Elevated heart rate in cardiovascular diseases: a target for treatment? Prog Cardiovasc Dis. 2009;52(1):46-60.

8. Fox K, Borer JS, Camm AJ, Danchin N, Ferrari R, Lopez Sendon JL, et al. Resting heart rate in cardiovascular disease. J Am Coll Cardiol. 2007;50(9): 823-30

9. Abbott TE, Ackland GL, Archbold RA, Wragg A, Kam E, Ahmad T, et al. Preoperative heart rate and myocardial injury after non-cardiac surgery: results of a predefined secondary analysis of the VISION study. $\mathrm{Br} J$ Anaesth. 2016:117(2):172-81

10. Levine GN, Bates ER, Bittl JA, Brindis RG, Finn SD, Fleisher LA, et al. 2016 ACC/AHA Guideline Focused Update on Duration of Dual Antiplatelet Therapy in Patients With Coronary Artery Disease: A Report of the American College of Cardiology/American Heart Association Task Force on Clinical Practice Guidelines: An Update of the 2011 ACCF/AHA/SCAI Guideline for Percutaneous Coronary Intervention, 2011 ACCF/AHA Guideline for Coronary Artery Bypass Graft Surgery, 2012 ACC/AHA/ACP/AATS/PCNA SCAI/STS Guideline for the Diagnosis and Management of Patients With Stable Ischemic Heart Disease, 2013 ACCF/AHA Guideline for the Management of ST-Elevation Myocardial Infarction, 2014 AHA/ACC Guideline for the Management of Patients With Non-ST-Elevation Acute Coronary Syndromes, and 2014 ACC/AHA Guideline on Perioperative Cardiovascular Evaluation and Management of Patients Undergoing Noncardiac Surgery. Circulation. 2016;

11. Abbott TEF, Pearse RM, Archbold RA, Wragg A, Kam E, Ahmad T, et al. Association between preoperative pulse pressure and perioperative myocardial injury: an international observational cohort study of patients undergoing noncardiac surgery. Br J Anaesth. 2017;1 19(1):78-86.

12. Boffa DJ, Allen MS, Grab JD, Gaissert HA, Harpole DH, Wright CD. Data from the Society of Thoracic Surgeons general thoracic surgery database: the surgical management of primary lung tumors. J Thorac Cardiovasc Surg. 2008;135(2):247-54.
13. Nojiri T, Inoue M, Takeuchi Y, Maeda H, Shintani Y, Sawabata N, et al. Impact of cardiopulmonary complications of lung cancer surgery on long-term outcomes. Surg Today. 2015;45(6):740-5.

14. Asakura K, Mitsuboshi S, Tsuji M, Sakamaki H, Otake S, Matsuda S, et al. Pulmonary arterial enlargement predicts cardiopulmonary complications after pulmonary resection for lung cancer: a retrospective cohort study. J Cardiothorac Surg. 2015;10:113.

15. Hammill BG, Curtis LH, Bennett-Guerrero E, O'Connor CM, Jollis JG, Schulman $\mathrm{KA}$, et al. Impact of heart failure on patients undergoing major noncardiac surgery. Anesthesiology. 2008;108(4):559-67.

16. Nanchen D, Leening MJ, Locatelli I, Cornuz J, Kors JA, Heeringa J, et al. Resting heart rate and the risk of heart failure in healthy adults: the Rotterdam study. Circ Heart Fail. 2013;6(3):403-10.

17. Butler J, Kalogeropoulos A, Georgiopoulou V, Belue R, Rodondi N, Garcia M, et al. Incident heart failure prediction in the elderly: the health $A B C$ heart failure score. Circ Heart Fail. 2008;1(2):125-33.

18. Auerbach AD, Goldman L. Beta-blockers and reduction of cardiac events in noncardiac surgery: scientific review. JAMA. 2002;287(11):1435-44.

19. Shammash JB, Trost JC, Gold JM, Berlin JA, Golden MA, Kimmel SE. Perioperative beta-blocker withdrawal and mortality in vascular surgical patients. Am Heart J. 2001;141(1):148-53.

20. Giles JW, Sear JW, Foex P. Effect of chronic beta-blockade on peri-operative outcome in patients undergoing non-cardiac surgery: an analysis of observational and case control studies. Anaesthesia. 2004;59(6):574-83.

21. Sander O, Welters ID, Foex P, Sear JW. Impact of prolonged elevated heart rate on incidence of major cardiac events in critically ill patients with a high risk of cardiac complications. Crit Care Med. 2005;33(1):81-8. discussion 241-2

22. Landesberg $\mathrm{G}$. The pathophysiology of perioperative myocardial infarction: facts and perspectives. J Cardiothorac Vasc Anesth. 2003;17(1):90-100.

23. Reich DL, Bennett-Guerrero E, Bodian CA, Hossain S, Winfree W, Krol M. Intraoperative tachycardia and hypertension are independently associated with adverse outcome in noncardiac surgery of long duration. Anesth Analg. 2002:95(2):273-7. table of contents

24. Hartmann B, Junger A, Rohrig R, Klasen J, Jost A, Benson M, et al. Intraoperative tachycardia and peri-operative outcome. Langenbeck's Arch Surg. 2003;388(4):255-60.

25. Parker MM, Shelhamer JH, Natanson C, Alling DW, Parrillo JE. Serial cardiovascular variables in survivors and nonsurvivors of human septic shock: heart rate as an early predictor of prognosis. Crit Care Med. 1987;15(10):923-9.

26. Pfister R, Michels G, Sharp SJ, Luben R, Wareham NJ, Khaw KT. Resting heart rate and incident heart failure in apparently healthy men and women in the EPIC-Norfolk study. Eur J Heart Fail. 2012;14(10):1163-70.

27. Kato Y, Suzuki S, Uejima T, Semba H, Nagayama O, Hayama E, et al. The relationship between resting heart rate and peak VO2: a comparison of atrial fibrillation and sinus rhythm. Eur J Prev Cardiol. 2016;23(13):1429-36.

Ready to submit your research? Choose BMC and benefit from:

- fast, convenient online submission

- thorough peer review by experienced researchers in your field

- rapid publication on acceptance

- support for research data, including large and complex data types

- gold Open Access which fosters wider collaboration and increased citations

- maximum visibility for your research: over $100 \mathrm{M}$ website views per year

At $\mathrm{BMC}$, research is always in progress.

Learn more biomedcentral.com/submissions 